\title{
End-to-side nerve flap for treatment of painful neuroma: A 15-year follow-up
}

\author{
LIZ ASHLEY, DO \\ JAMES O. STALLINGS, MD
}

Traumatic neuromas are notorious for the rate at which they recur after excision. A method involving resection of the neuroma, creation of a proximal endto-side nerve flap, and burial of the flap away from the neuroma site and under muscles is described. The end-to-side nerve flap and the muscle provide an environment similar anatomically and physiologically to that of the nerve ending site, away from tension, scar tissue, and trauma. In the case reported this technique was carried out in the dorsal sensory branch of the left ulnar nerve, with placement of the nerve end in the belly of the flexor carpi ulnaris muscle. The patient returned promptly to work and has been free of pain for 15 years. Similar excellent results have been achieved in five other cases followed from 7 to 12 years.

Painful neuromas are notoriously difficult to treat and recur with displeasing frequency. To date numerous treatments have been attempted, but success rates are far from high. We present an alternative method, which in six cases has been effective in relieving troublesome symptoms. The technique involves resection of the neuroma and creation of a proximal end-to-side nerve flap, which is buried away from the resected neuroma site under a large protective structure free from scar tissue, most often a muscle belly. The following is a report of the first case, for which the follow-up time is 15 years.

\section{Report of case}

A 22-year-old grocery clerk was referred for evaluation and treatment of a refractory, painful neuroma of the dorsal sensory branch of the left ulnar nerve in August 1972. He had been involved in a motorcycle accident in August 1967, resulting in a laceration of the distal part of the forearm and wrist that severed the extensor carpi ulnaris tendon in two places at the wrist. No nerve damage was diagnosed when the tendons were repaired primarily by another surgeon. Over the next two years, the painful neuroma occurred along with local abscess formation. This abscess formation was from the sutures that were used to repair the tendon of the flexor carpi ulnaris. The abscess was drained, and several old retained sutures were removed. Five years after the original injury, the neuroma was excised and additional retained sutures removed by another surgeon. Because of significant residual pain, procaine injections were given repeatedly but failed to provide lasting relief.

Tenderness remained along the medial aspect of the ulna distally. Loss of sensation was found distally in the distribution of the dorsal sensory branch of the ulnar nerve. No motor deficits or trophic changes were noted. The patient was severely disabled: He could not work, make a fist, nor bear to have a sleeve touch his wrist.

By the time we saw him he had undergone four operations and numerous injections. Our exploration showed a very large, recurrent neuroma of the dorsal branch of the left ulnar nerve located just proximal to the pisiform bone. Considerable scar tissue, many old silk sutures, and silicone that had been used to cap the nerve were present. Because of the high recurrence rate of neuroma after excision, it was thought that an end-toside nerve flap after excision might minimize his symptoms. This is precisely what we did. The flap was placed beneath the belly of the flexor carpi ulnaris muscle well away from the original site of injury.

Fifteen years later the patient remains free from pain. $\mathrm{He}$ has complete range of motion, full strength, and entire use of his left hand and arm. He has been able to 
be gainfully employed as of three months after his surgery in 1972 .

\section{Operative technique}

An elliptical incision was made along the ulnar aspect of the left wrist extending into the forearm. As much old scar tissue and retained old silk sutures were removed as possible. The main ulnar nerve in the distal part of the forearm under the flexor carpi ulnaris muscle was dissected free and the division into the dorsal sensory branch of the ulnar nerve was identified with its neuromas surrounded by and adhered to scar tissue and bone (Fig 1). Neurolysis and decompression of the main part of the ulnar nerve was done under magnification at this level. The dissection included complete freeing of the ulnar nerve and its dorsal sensory branch proximally and distally away from the scarred area. The distal offshoots of the dorsal sensory branch were placed on traction and then cut and allowed to retract back into an area free from scar tissue. Proximally, the neuroma was excised.

A site precisely $6 \mathrm{~cm}$ proximal to the ulnar styloid process was chosen for the nerve flap placement to insure its remoteness from the scarred area and its protection from local injury by being deep to a muscle belly. With allowance for a $1 \mathrm{~cm}$ nerve flap loop, an outer epineurial flap was elevated 6 $\mathrm{cm}$ proximal to the ulnar styloid process on the dorsal sensory branch of the ulnar nerve so that an epineurial defect or "window" was created. Special care was taken not to disrupt any perineurium. Two $\mathrm{cm}$ distal to this window the dorsal sensory branch of the ulnar nerve was transected by sharp dissection. The end was folded back upon itself and positioned to fill the epineurial defect. Excess epineurial flap was trimmed and discarded.

An end-to-side suturing of epineurium to epineurium was performed with 8-0 nylon under $25 \times$ magnification (Fig 2). Approximately ten sutures were placed so as to completely cover the raw ends with epineurium and intact perineurium and fold the nerve back on itself without tension. Its final position was located deep to the wrist flexor ulnar muscle belly. The subcutaneous tissues and skin were closed and a cigarette drain was placed. A splint and pressure dressings were applied with the wrist in neutral position.

\section{Other clinical experience}

This end-to-side flap has been used in five other cases. Two involved the superficial branch of the radial nerve; the end-to-side nerve flap was buried beneath the brachioradialis muscle. In three instances involving the dorsal sensory branch of the ulnar nerve, end-to-side nerve flap was buried be-

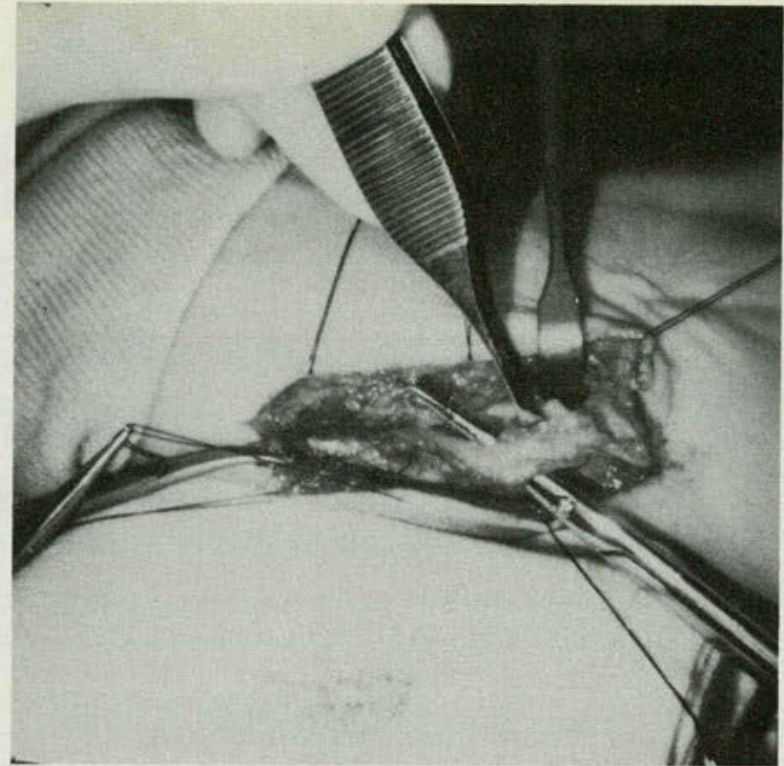

Fig 1. Operative dissection showing neuroma.

neath the ulnar flexor muscle of the wrist. The results in these five cases have been excellent, with the patients free of pain. Follow-up times vary from 7 to 12 years.

\section{Review of the literature}

A traumatic neuroma is a focal mass on a peripheral nerve that results from local injury. ${ }^{1}$ The gross appearance is that of a swollen, firm nerve. Microscopically, one sees "a proliferating coil of neuraxons and Schwann cells" interlaced with the scar of repair. ${ }^{2}$ A disorganized pattern of the nerve is embedded in a dense stroma of connective tissue. ${ }^{3}$

Most neuromas are asymptomatic. ${ }^{4}$ When painful, symptoms vary widely. The neuroma may persist unaltered for years or grow progressively. Pain may be dull, aching, piercing, spontaneous, or triggered. Usually, a trigger area can be identified. ${ }^{5}$ Often, Tinel's sign is the earliest indication of its presence. ${ }^{6}$ The size of a neuroma does not necessarily correlate with its ability to produce symptoms. ${ }^{7}$

In peripheral nerves after injury, the regenerating axons' capacity for growth is unlimited provided that the neuron's central cell body remains intact. Thus, the proximal end of a severed nerve fiber will grow until it reaches an end organ or growth is restricted by some other means. ${ }^{8}$

Sunderland $^{9}$ stated that undamaged perineurium surrounding each funiculus presents a barrier to passage of regenerating axons; thus, if the damaged funiculi could be sealed, axonal escape 


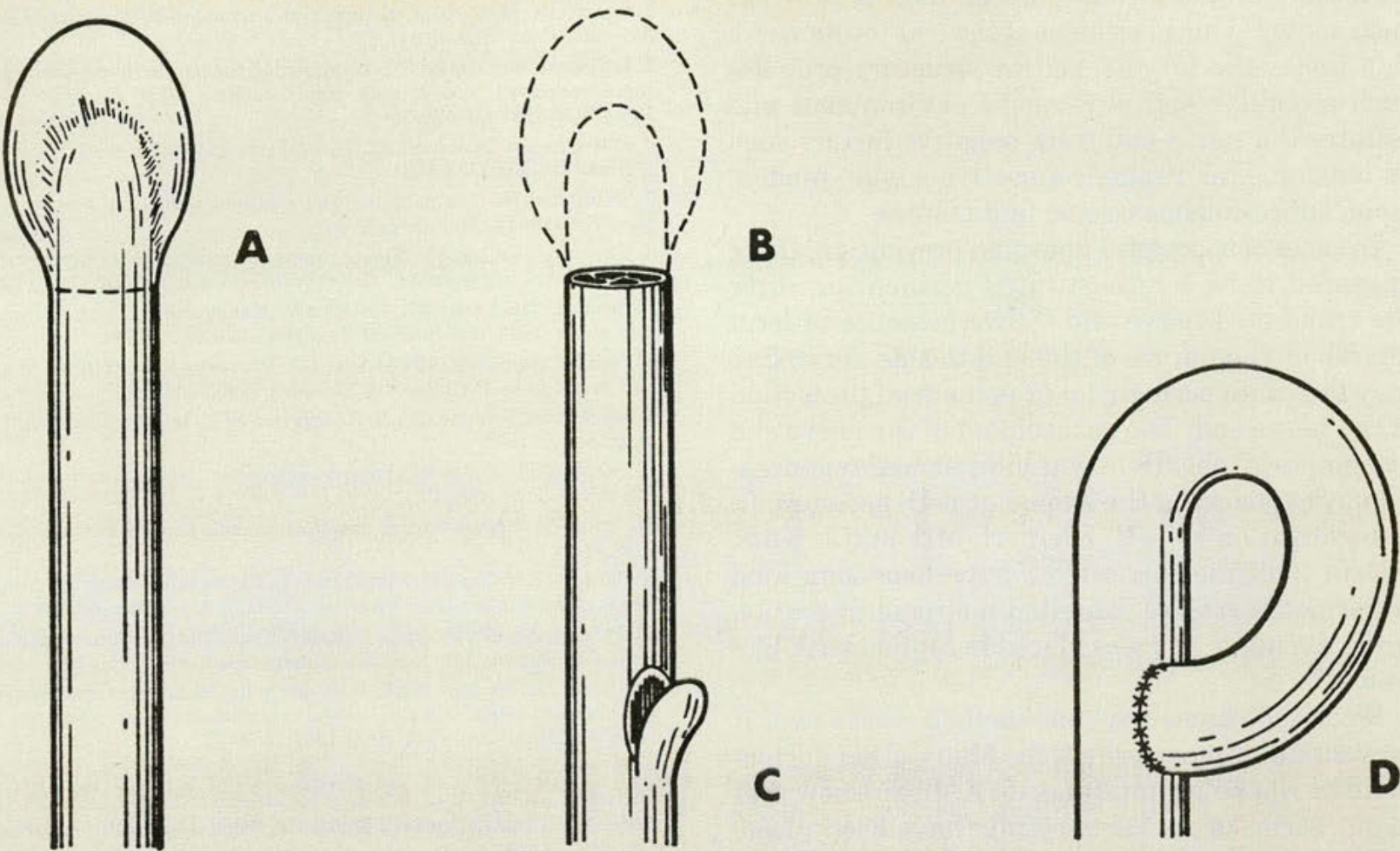

Fig 2. Epineurial nerve flap. A, Terminal bulbous neuroma. B, Complete sharp transection of neuroma. C, "Window" created by elevation of outer epineurial flap. D, Transected end folded back upon itself, placed into window defect, and sutured.

could be prevented and growth of neuroma forestalled. It has been clinically observed that a well sealed epineurial repair of a transected peripheral nerve seldom is followed by development of a painful neuroma. These observations suggest that containment of the regenerating axons within intact epineurium or perineurium might prevent tenderness. ${ }^{9}$

Clean division of a nerve trunk with a sharp knife so that it will retract into soft tissue environment away from trauma, weight-bearing, or scarring ${ }^{9}$ is recommended for avoiding neuroma formation. ${ }^{4}$ Tension with its concurrent increase in scar formation is also thought to play an important role in painful neuroma formation, so undue tension on the injured nerve should be avoided. ${ }^{3}$

Many ingenious methods for treatment and prevention of painful neuroma have been devised. These include methods to suppress axon regeneration or restrict growth of axons and include physical and chemical methods. Such treatment has not been completely satisfactory. ${ }^{9}$

Several authors ${ }^{9-13}$ have reported absence of neuroma formation when transected nerves are embedded in muscle fibers. In 1918, Moskowicz ${ }^{10}$ treated two cases in this manner, believing that he could prevent further neuroma formation, though no histologic results were offered. In 1949 , Teneff ${ }^{11}$ reported absence of amputation neuroma formation in experimental rabbit studies after placing principal nerves, unsutured, between muscle fibers near the amputation site. Histologic studies showed physiologic connections between the terminal neurofibrils and the muscle fibers. In 1959 , Munro and Mallory ${ }^{12}$ reported success with a combination of resection of the neuroma and crushing and ligating the transected nerve ending, and placement in red muscle fibers. Petropoulos and Stefanko, ${ }^{13}$ reported absence of neuroma formation in a dog after transected frayed nerve fibers were implanted into muscle.

More recently Dellon and Mackinnon ${ }^{3}$ reported results in a series of cases treated with muscle implantation.

\section{Discussion}

The principle in reconstructive plastic surgery that one should reconstruct with like tissues if at all possible is well established. Our original thought was that surrounding the freshly cut nerve end 
with like tissues would probably minimize symptomatic neuroma formation. Red muscle provides such a site. ${ }^{11}$ Our placement of the end-to-site nerve flap under the largest, softest structure provides such a similar and physiologic environment and isolates the nerve end from negative factors such as tension, scar tissue, connective tissue, tendon, bone, subcutaneous tissue, and trauma.

In cases of successful neuroma prevention, there appeared to be a reconstituted perineurium over the transected nerve end. ${ }^{14}$ The presence of local muscle or the nature of the end-to-side nerve flap may facilitate perineurial or epineurial protection of the nerve end. The encasement of the nerve end by epineurial sheath may inhibit axonal regeneration by overcoming the axonal growth pressure. In a conversation with $\mathrm{H}$. Kleinert, MD, and J. Kutz, $\mathrm{MD}$, in 1983, they indicated that perhaps some kind of hormonal release inhibited neuroma formation when the nerve end was placed in contact with like tissue.

We do not know why our method works or if it prevents neuroma formation. Many other factors could be working simultaneously. We do know that in our series of six cases results have been pleasing in that all the patients are pain free and have returned promptly to work.
1. Spencer PS: The traumatic neuroma and proximal stump. Bull Hosp Jt Dis 1974;35:85-102.

2. Grant GH: Methods of treatment of neuromata of the hand. $J$ Bone Joint Surg Am 1951;33:841-848.

3. Dellon Al, Mackinnon SE: Treatment of the painful neuroma by neuroma resection and muscle implantation. Plast Reconstr Surg 1986;77(March):427-438.

4. Mathews GJ, Osterholm JL: Painful traumatic neuromas. Surg Clin N Amer 1972;51:1313-1324.

5. Williams HB: The painful stump neuroma and its treatment. Clin Plast Surg1984;11(January):79-84.

6. Kline DG, Nulsen FE: The neuroma in continuity: Its preoperative and operative management. Surg Clin North Am 1972;52:1189-1209.

7. Herndon JH, Eaton RG, Littler JW: Management of painful neuromas in the hand. $J$ Bone Joint Surg Am 1976;58:369-373.

8. Poth EJ, Fernandez EB, Drager GA: Prevention of formation of endbulb neuromata. Proc Soc Exp Biol Med 1945;60:200-207.

9. Sunderland S: Nerve and Nerve Injuries, ed. 2. London, Churchill Livingstone $\mathrm{Co}, 1978$.

10. Moskowiz L: Zur Behandlung der schmerzhaften Neurome. Zentralbl Chir 1918;45:(No 32)547.

11. Teneff S: Prevention of amputation neuroma. $J$ Int Coll Surg 1949;12:16-20.

12. Munro D, Mallory K: Elimination of the so-called amputation neuromas of divided peripheral nerves. $N$ Engl $J$ Med 1959;260:358-361.

13. Petropoulos PC, Stefanko S: Experimental observations on the prevention of neuroma formation: Preliminary report. J Surg Res 1961;1:241.

14. Dellon AL, Mackinnon SE, Pestronk A: Implantation of sensory nerve into muscle: Preliminary clinical and experimental observations on neuroma formation. Ann Plast Surg 1984;12:30-40.

From the Plastic Surgery Institute, West Des Moines, Iowa.

Reprint request to Dr Stallings, Plastic Surgery Institute, 1025 Ashworth Rd, Suite 528, West Des Moines, IA 50268. 


\section{Introducing a Double Sirength tablet to subdue pain/inflammation}



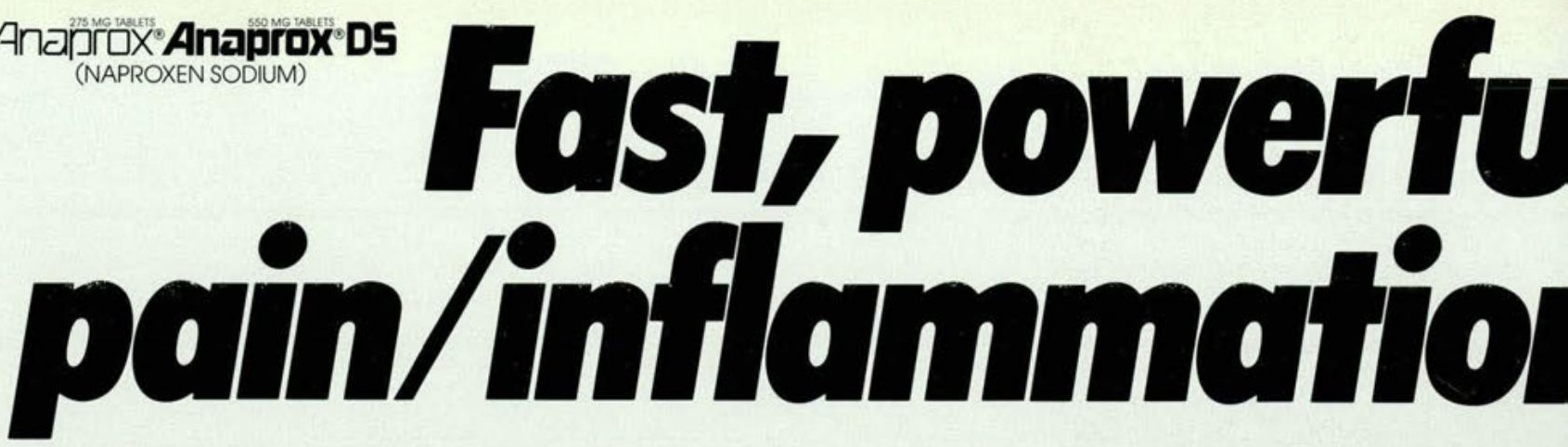

Now, there is ANAPROX Double Strength that gives you more analgesic/anti-inflammatory activity per dose. It joins the proven ANAPROX $275 \mathrm{mg}$ strength to provide you with greater dosage flexibility and convenience.

Twice the strength-550 mg ANAPROX per tablet.

- Dosage- -10 The daily dosage of $1650 \mathrm{mg}$ can be used for limited periods when a higher level of analgesic/anti-inflammatory activity is required. Do not exceed this daily dose.

- Greater analgesic/anti-inflammatory activity. in each tablet-reduces inflammation as it relieves the pain.

- No oral analgesic works faster-onset of pain relief may occur as fast as 20 minutes.

- Efficacy equal to the narcotic combinationseffective analgesia with a potent anti-inflammatory bonus the codeine combinations and propoxyphene napsylate can't offer.

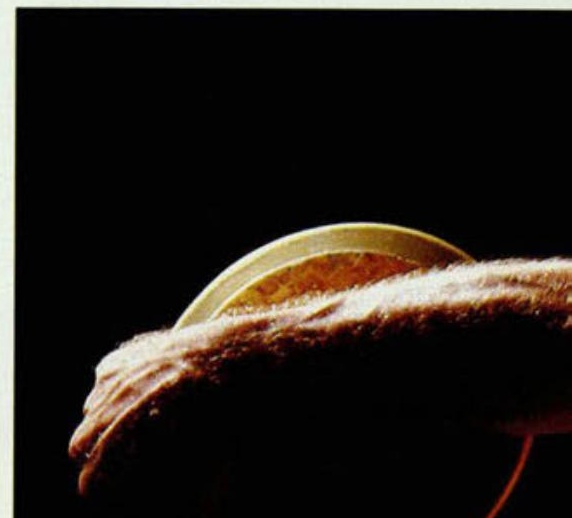

Non-narcotic-no narcotic-type side effects* or clinical limitations. 


\section{Fast, powerful relief for the pain inflammation you see every day}
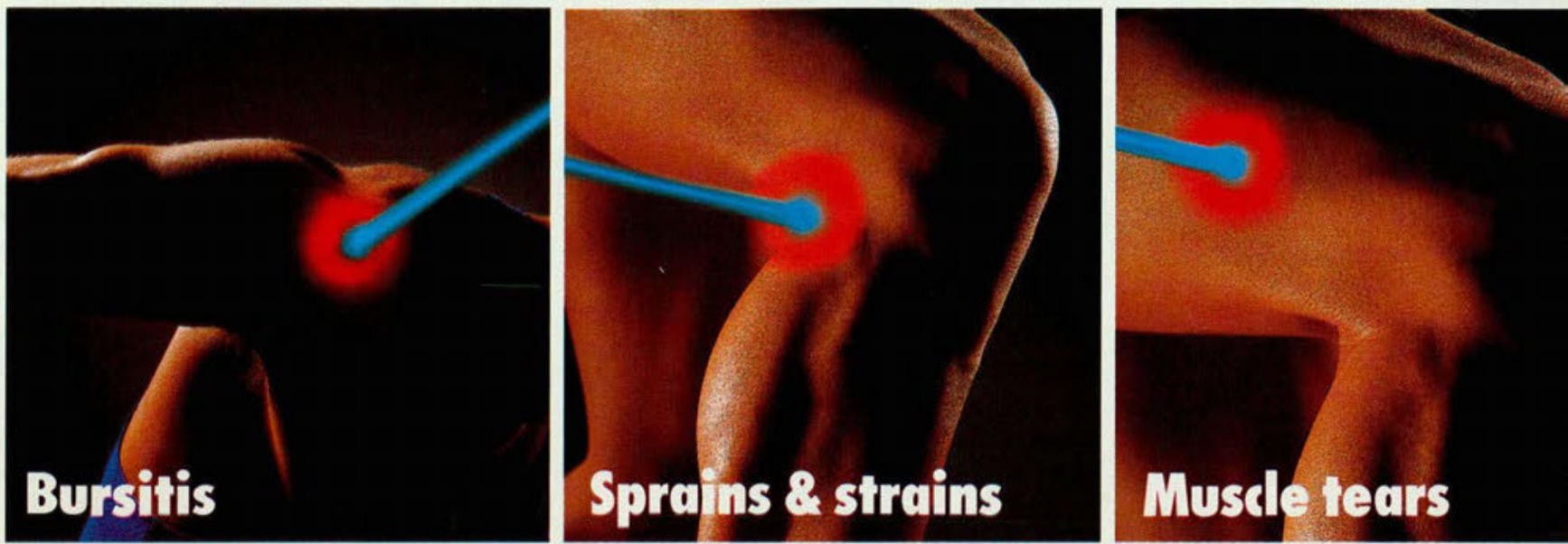

\section{Musculoskeletal trauma}

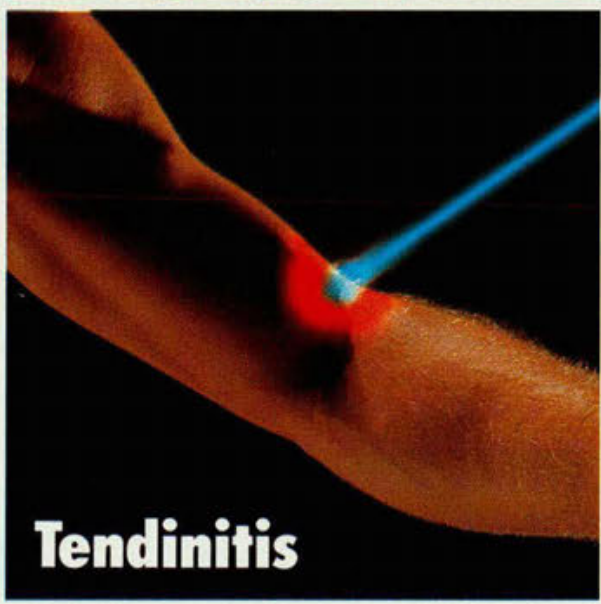

ANAPROX ${ }^{\circ}$ and ANAPROX DS (naproxen sodium) Tablets

Brief Summary:

Indications: Relief of mild to moderate pain; treatment of primary dysmenorrhea Treatment of rheumatoid arthritis, ostearrthritis, juvenile arthritis, ankylosing spondylitis, tendinitis and bursitis, and acute gout Contraindications: Patients who have had allergic reactions to NAPROSYN. ANAPROX or ANAPROX DS or in whom aspirin or other NSAIDs induce the syndrome of asthma, rhinitis, and nasal polyps. Because anaphylactic reactions usually occur in patients with a history of such reactions, question patients for
asthma, nasal polyps, urticaria, and hypotension associated with NSAIDs asthma, nasal polyps, urticaria, and hypotension associated with NS
before starting therapy. If such symptoms occur, discontinue the drug. Warnings: Peptic ulcers and GI bleeding have been reported in patients on NSAIDs, including naproxen sodium. In patients with GI bleeding or active pe tic ulcer, start an anti-ulcer regimen, weigh benefits and risks of treatment, and monitor patient. Give to patients with history of Gl disease only under close supervision and after reading Adverse Reactions section

Precautions: DO NOT GIVE NAPROSYNo (NAPROXEN) CONCOMITANTIY WITH ANAPROX OR ANAPROX OS (NAPROXEN SODIUM SINCE BOTH CIRCULATE IN PLASMA AS THE NAPROXEN ANION. Acute interstitial nephritis with hematuria, proteinuria, and nephrotic syndrome has been reported. Patients with ria, proteinuria, and nephrotic syndrome has been reported. Patients with
impaired renal function, heart faliure, liver dystunction, taking diuretics, and impaired renal function, heart taliure, liver dystunction, taking diuretics, and
the elderly are at greater risk of overt renal decompensation. If this occurs, discontinue the drug Use with caution and monitor serum creatinine and/or creat nine clearance in patients with significantly impaired renal function. Use caution in patients with baseline creatinine clearance less than $20 \mathrm{~m} /$ minute Use the lowest effective dose in the elderly or in patients with chronic alcoholic liver disease or cirrhosis. With NSAIDs, borderline elevations of liver tests may occur in up $10.5 \%$ of patients They may progess, occur in up to $15 \%$ of patients. They may progress, remain unchanged, or be

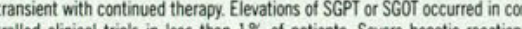
including jaundice and tatal hepatitis, have been reported rarely. If liver disease including jaundice and fatal hepatitis, have been reported rarely. If Iiver disease
develops or if systemic manifestations occur (e.g., eosinophilia or rashi), discon-

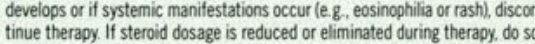
slowly and observe patients closely for adverse effects, including adrenal insulficiency and exacerbation of arthritis symptoms. Determine hemioglobin values periodically for patients with initial values of 10 grams or less who receive long term therapy. Peripheral edema has been reported. For patients with restricted

sodium intake, note that each tablet contains approximately $25 \mathrm{mg}$ or $50 \mathrm{mg}$ (1 or $2 \mathrm{mEq}$ ) sodium. Use with caution in patients with fluid retention, hypertension or heart failure. The drug's antipyretic and anti-inflammatory activities may reduce fever and inflammation, diminishing their diagnostic value. Conduct ophthalmic studies if any change or disturbance in vision occurs. Information for Patients: Patients should use caution for activities requir. ing alertness if they experience drowsiness, diziness, vertigo or depression during therapy

Drug Interactions: Use caution when giving concomitantly with coumarin. Orug Interactions: Use caution when giving concomitantly with coumarintype anticaagulants; a hydantoin, sulfonamide or sufor

ium; beta-blockers; probenecid; or methotrexate.
Drug/Laboratory Test Interactions: The drug may decrease platelet aggregation and prolong bleeding time or increase urinary values for 17 -ketogenic steroids. Temporarily stop therapy for 72 hours before doing adrenal function tests. The drug may interfere with urinary assays of 5 HIAA.

Carcinogenesis: A 2-year rat study showed no evidence of carcinogenicity. Pregnancy: Category B. Do not use during pregnancy unless clearly needed. Avoid use during late pregnancy.

Nursing Mothers: Avoid use in nursing mothers.

Pediatric Use: Single doses of 25-5 mg/kg (as naproxen suspension), with total daily dose not exceeding $15 \mathrm{mg} / \mathrm{kg} /$ day, are safe in children over 2 years of age

Adverse Reactions: In a study, GI reactions were more frequent and severe in rheumatoid arthritis patients on $1500 \mathrm{mg} /$ day naproxen than in those on 750 $\mathrm{mg} / \mathrm{day}$ (doses comparable to $1650 \mathrm{mg}$ and $825 \mathrm{mg}$ of naproxen sodium). In studies in children with juvenile arthritis, rash and prolonged bleeding times were more frequent, Gl and CNS reactions about the same, and other reactions less frequent than in adults. Incidence Greater Than 1\%. Probable Causal Relationship: Gl: The most frequent complaints related to the GI tract: constipation * heartburn" abdominal pain" nausea* dyspepsia, diarrhea, stomatitis. CNS. headache* diziness* drowsiness*" light-headedness, vertigo Derma. tologic itching (pruritus)" skin eruptions" ecchymoses" sweating. purpura. Special Senses: tinnitus," hearing disturbances, visual disturbances. Cardiovascular: edema*" dyspnea*" palpitations. General: thirst. "Incidence of reported reaction $3 \%-9 \%$. Where unmarked, incidence less than $3 \%$. Incidence Less Than 1\%: Probable Causal Relationship: Gl: abnormal liver function tests, colitis, GI bleeding and/or perforation, hematemesis, jaundice, melena. peptic ulceration with bleeding and/or perforation, vomiting. Renal: glomerular

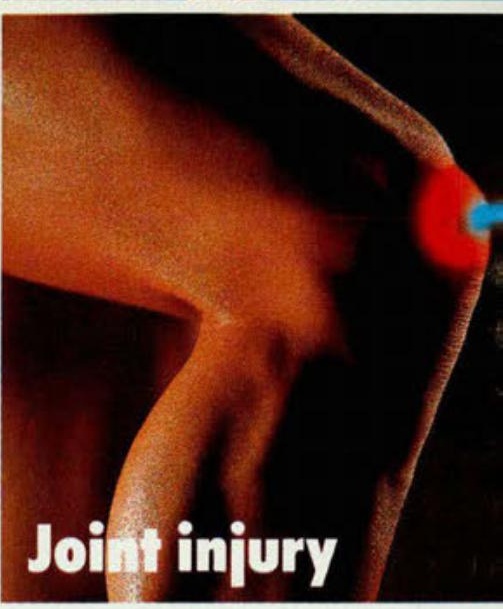

nephritis, hematuria, interstitial nephritis, nephrotic syndrome, renal dis Hematologic: agranulocytosis, eosinophilia, granulocytopenia, leukop thrombocytopenia. CNS: depression, dream abnormalities, inability to $\mathrm{co}$ trate, insomnia, malaise, myalgia and muscle weakness. Dermatologic: cia, photosensitive dermatitis, skin rashes. Special Senses: hea impairment Cardiovascular: congestive heart failure. Respiratory: eosinos pneumonitis. General: anaphylactoid reactions, menstrual disorders, py (chills and fever) Causal Relationship Unknown: Hematologic: aplastic an hemolytic anemia CNS: cognitive dysfunction. Dermatologic: epidermal Iysis, enthema multiforme, Stevens.Johnson syndrome, urticaria Gl: ulcer: stomatitis Cardiovascular: vasculitis, General: angioneurotic edema, glycemia, hypoglycemia

Overdosage: May have drowsiness, heartburn, indigestion, nausea, vom Empty stomach and use usual supportive measures. Prompt administrat 5 grams activated charcoal may reduce drug absorption. Hemodialysis ma appropriate in renal failure.

Dosage and Administration for Mild to Moderate Pain. Dysmenorrthe Acute Tendinitis and Bursitis: The recommended startiog dose is $550 \mathrm{~m}$ lowed by 275 mgevery 6 to 8 hours, as required. The total deily dose exceed $1375 \mathrm{mg}$. exceed $1375 \mathrm{mg}$

Dosaministration for Rheumatoid Arthritis, Osteoarthritis Ankylosing Spondylitis: The recommended dose in adults is $275 \mathrm{mg}$ or 55 twice daily In patients who tolerate lower doses well, the dose may be incre to $1,650 \mathrm{mg}$ per day for limited periods when a higher level of inflammatory/analgesic activity is required. At this dosage, physicians sh observe sufficient increased clinical benefits to offset potential increase Caution: Federal law prohibits dispensing without prescription. See package insert for full Prescribing Information. (c) Revised S SYNTEX

Introducin

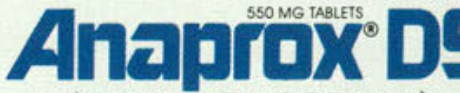

(NAPROXXEN SODIUM) 